TRANSACTIONS OF THE

AMERICAN MATHEMATICAL SOCIETY

Volume 356, Number 6, Pages 2139-2147

S 0002-9947(04)03159-9

Article electronically published on February 2, 2004

\title{
SUR LES TRANSFORMÉES DE RIESZ DANS LE CAS DU LAPLACIEN AVEC DRIFT
}

\author{
NOËL LOHOUÉ AND SAMI MUSTAPHA
}

Abstract. We prove $L^{p}$ estimates for Riesz transforms with drift.

\section{INTRODUCTION}

La bornitude des transformées de Riesz $\frac{\partial}{\partial x_{j}} \Delta^{-1 / 2}, 1 \leq j \leq n$, sur les espaces $L^{p}$, $1<p<\infty$, dans $\mathbb{R}^{n}$ a été étendue, grâce à la théorie des intégrales singulières dans les espaces de type homogène (cf. 4]) à d'autres contextes où le volume des boules croît d'une manière polynomiale (ex. variétés à courbure positive, groupes de Lie à croîssance polynomiale, groupes discrets nilpotents, etc.; cf. [1], [2]). Dès que l'on se trouve en présence d'une croîssance superpolynomiale du volume l'étude de la bornitude des transformées de Riesz devient problématique. L'investigation de ce type de situations a fait l'objet de plusieurs travaux de la part du premier auteur (cf. par exemple 7 pour les groupes de Lie non moyennables et 8 pour les variétés Riemanniennes à courbure minorée). D'autres résultats ont été obtenus récemment pour les groupes de Lie moyennables non-unimodulaires et pour certains espaces homogènes avec une croissance du volume exponentielle (cf. 9]).

Il existe néanmoins un contexte élémentaire où le problème de Riesz se pose d'une manière naturelle en présence d'un volume exponentiel. En effet, en se plaçant dans l'espace euclidean $\mathbb{R}^{n}$ et en ajoutant au Laplacien standard un "Drift" on obtient un opérateur qui est auto-adjoint pour une mesure pour laquelle la croissance du volume des boules est exponentielle. L'investigation de la bornitude des transformées de Riesz sur les espaces $L^{p}$ correspondant à cette mesure ne peut plus se faire via la théorie de Calderon-Zygmund.

Nous étudions ci-dessous ce problème. La méthode que nous utilisons, qui est basée sur l'utilisation du noyau de la chaleur, se généralise très naturellement dans le contexte des sous-Laplaciens sur les groupes de Lie moyennables. Elle permet d'obtenir dans ce contexte un certain nombre de résultats concernant la bornitude des transformées de Riesz pour des sous-Laplaciens avec Drift. Nous illustrons ces résultats à travers l'exemple du groupe de Heisenberg et de certains groupes résolubles de rang 1.

Received by the editors October 30, 1998.

2000 Mathematics Subject Classification. Primary 58Jxx, 43-XX; Secondary 35Jxx, 35Kxx.

Key words and phrases. Riesz transforms, drift, Lie groups, convolution. 


\section{ENONCÉ DE RÉSULTATS}

Considérons dans $\mathbb{R}^{n}$ l'opérateur $L=\Delta+X$ où $\Delta$ est le Laplacien sandard et où $X$ est un "Drift" donné par $X=\sum_{i=1}^{x} c_{j} \partial_{x_{j}}, c_{j} \in \mathbb{R}, 1 \leq j \leq n$. Notons $\varphi(x)=\exp \left[\frac{1}{2} \sum_{j=1}^{n} c_{j} x_{j}\right]$ et $\lambda=\frac{1}{4} \sum_{j=1}^{n} c_{j}^{2}$. Nous supposerons dans toute la suite que $\lambda \neq 0$ (i.e. qu'il existe réellement un Drift dans l'opérateur $L$ ). On vérifie (cf. §III, ci-dessous) que l'opérateur $L$ est auto-adjoint sur l'espace de Hilbert $L^{2}\left(\mathbb{R}^{n}, \varphi^{-2} d x\right)$, où $d x$ désigne la mesure de Lebesgue sur $\mathbb{R}^{n}$ et que

$$
\inf \left\{\langle L f, f\rangle,\|f\|_{L^{2}\left(\varphi^{-2} d x\right)}=1\right\}=\lambda,
$$

où $\langle\cdot, \cdot\rangle$ désigne le produit scalaire dans $L^{2}\left(\varphi^{-2} d x\right)$. Soit $0 \leq \theta \leq 1$, posons $\mathcal{L}_{\theta}=L-\theta \lambda$. Soit $\mathcal{L}_{\theta}=\int_{(1-\theta) \lambda}^{\infty} \mu d E_{\mu}$ la décomposition spectrale de $\overline{\mathcal{L}}_{\theta}$ (cf. [13]). Notons $\mathcal{L}_{\theta}^{-k / 2}=\int_{(1-\theta \lambda)}^{\infty} \mu^{-k / 2} d E_{\mu}, k=1,2, \ldots$ Alors:

Théorème 1. Les notations étant comme ci-dessus, on a:

(i) Soit $0<\theta<1$. Alors pour tout $p$ vérifiant

$$
\frac{2}{1+\sqrt{1-\theta}}<p<\frac{2}{1-\sqrt{1-\theta}}
$$

pour tout $k=1,2, \ldots$ et pour tout $1 \leq i_{1}, i_{2}, \ldots, i_{k} \leq n$ la transformée de Riesz multiple $\partial_{x_{i_{k}}} \cdots \partial_{x_{i_{2}}} \partial_{x_{i_{k}}} \mathcal{L}_{\theta}^{-k / 2}$ est bornée de $L^{p}\left(\varphi^{-2} d x\right)$ dans $L^{p}\left(\varphi^{-2} d x\right)$. De plus si les coefficients $c_{i_{k}}$ sont tous non nuls et si $p \leq 2 /(1+\sqrt{1-\theta})$ ou $p \geq 2 /(1-\sqrt{1-\theta})$ alors $\partial_{x_{i_{k}}} \cdots \partial_{x_{i_{2}}} \partial_{x_{i_{k}}} \mathcal{L}_{\theta}^{-k / 2}$ est non bornée de $L^{p}\left(\varphi^{-2} d x\right)$ dans $L^{p}\left(\varphi^{-2} d x\right)$.

(ii) Si $\theta=0$ alors pour tout $k=1,2, \ldots$ et pour tout $1 \leq i_{1}, i_{2}, \ldots, i_{k} \leq n$ l'opérateur $\partial_{x_{i_{k}}} \cdots \partial_{x_{i_{2}}} \partial_{x_{i_{k}}} \mathcal{L}_{\theta}^{-k / 2}$ est borné sur $L^{p}\left(\varphi^{-2} d x\right)$ pour tout $1<p<\infty$.

(iii) Si $\theta=1$ et si les coefficients $c_{i_{k}}$ sont tous non nuls $\partial_{x_{i_{k}}} \cdots \partial_{x_{i_{2}}} \partial_{x_{i_{k}}} \mathcal{L}_{\theta}^{-k / 2}$ est non borné sur $L^{p}\left(\varphi^{-2} d x\right), \forall 1<p<\infty$.

Le théorème ci-dessus se généralise très naturellement dans le contexte des groupes de Lie. Soit $G$ un groupe de Lie connexe moyennable et soient $X_{1}, \ldots, X_{n}$ des champs de vecteurs invariants à gauche sur $G$ vérifiant la condition de Hörmander (cf. [12]). Soit $\Delta=-\sum X_{j}^{2}$ le sous-Laplacien qui leur correspond. Soit $L$ l'opérateur défini par:

$$
L=\Delta+\sum_{j=1}^{n} c_{j} X_{j}=-\sum_{j=1}^{n} X_{j}^{2}+\sum_{j=1}^{n} c_{j} X_{j}
$$

où les cofficients $c_{j} \in \mathbb{R}$ ne sont pas tous nuls. On fait l'hypothèse suivante:

$$
\left\{\begin{array}{l}
\text { Il existe un caractère } \chi \text { sur } G \text { et il existe } \lambda>0 \text { telle que } \\
L=\chi(\Delta+\lambda) \chi^{-1} .
\end{array}\right.
$$

Il est facile de voir que si un tel caractère existe, alors nécessairement

$$
X_{j}(\chi)=\frac{c j}{2} \chi, \quad 1 \leq j \leq n,
$$

et

$$
\lambda=\frac{1}{4} \sum_{j=1}^{n} c_{j}^{2} .
$$


On vérifie alors que l'opérateur $L$ est auto-adjoint sur $L^{2}\left(\chi^{-2} d^{r} g\right)$ où $d^{r} g$ est la mesure de Haar invariante à droite sur $G$ et que

$$
\inf \left\{\int L f \cdot f \chi^{-2} d^{r} g,\|f\|_{L^{2}\left(\varphi^{-2} d^{r} g\right)}=1\right\}=\lambda .
$$

On note, comme ci-dessus, pour $0 \leq \theta \leq 1, \mathcal{L}_{\theta}=L-\theta \lambda$ et on considère $\mathcal{L}_{\theta}^{-k / 2}=$ $\int_{(1-\theta \lambda)}^{\infty} \mu^{-k / 2} d E_{\mu}, k=1,2, \ldots$, où $\mathcal{L}_{\theta}=\int_{(1-\theta) \lambda}^{\infty} \mu d E_{\mu}$ est la décomposition spectrale de $\mathcal{L}_{\theta}$.

Théorème 2. Soit $G$ un groupe de Lie connexe moyennable et soit $L=\Delta+X$ comme ci-dessus. Supposons l'hypothèse $(\mathrm{H})$ vérifiée. Alors

(i) Soit $0<\theta<1$. Alors pour tout $p$ vérifiant

$$
\frac{2}{1+\sqrt{1-\theta}}<p<\frac{2}{1-\sqrt{1-\theta}}
$$

pour tout $k=1,2, \ldots$ et pour tout $1 \leq i_{1}, i_{2}, \ldots, i_{k} \leq n$ la transformée de Riesz multiple $X_{i_{1}} \ldots X_{i_{k}} \mathcal{L}_{\theta}^{-k / 2}$ est bornée de $L^{p}\left(G, \chi^{-2} d^{r} g\right)$ dans $L^{p}\left(G, \chi^{-2} d^{r} g\right)$. De plus si les coefficients $c_{i_{k}}$ sont tous non nuls et si $p \leq 2 /(1+\sqrt{1-\theta})$ ou $p \geq$ $2 /(1-\sqrt{1-\theta}) X_{i_{1}} \ldots X_{i_{k}} \mathcal{L}_{\theta}^{-k / 2}$ est non bornée de $L^{p}\left(G, \chi^{-2} d^{r} g\right)$ dans $L^{p}\left(\chi^{-2} d^{r} g\right)$.

(ii) Si $\theta=0$ alors pour tout $k=1,2, \ldots$ et pour tout $1 \leq i_{1}, i_{2}, \ldots, i_{k} \leq n$ l'opérateur $X_{i_{1}} \ldots X_{i_{k}} \mathcal{L}_{\theta}^{-k / 2}$ est borné sur $L^{p}\left(G, \chi^{-2} d^{r} g\right)$ pour tout $1<p<\infty$.

(iii) Si $\theta=1$ et si les coefficients $c_{i_{k}}$ sont tous non nuls $X_{i_{1}} \ldots X_{i_{k}} \mathcal{L}_{\theta}^{-k / 2}$ est non borné $\operatorname{sur} L^{p}\left(G, \chi^{-2} d^{r} g\right), \forall 1<p<\infty$.

\section{Preuve DES THÉORÈMES}

III. 1. Dans $\mathbb{R}^{n}$ l'opérateur $L=-\sum_{j=1}^{n} \frac{\partial^{2}}{\partial x_{j}^{2}}+c_{1} \frac{\partial}{\partial x_{1}}+\cdots+c_{n} \frac{\partial}{\partial x_{n}}$ définit bien un opérateur auto-adjoint sur $L^{2}\left(\mathbb{R}^{n}, \varphi^{-2} d x\right)$. En effet, les notations étant les mêmes que dans $\S I I$, on a:

$$
\varphi \Delta\left(\varphi^{-1} f\right)=-\left(\frac{1}{4} \sum_{j=1}^{n} c_{j}^{2}\right) f+X f+\Delta f .
$$

D'où l'on déduit la relation de conjugaison

$$
L f=\varphi(\Delta+\lambda) \varphi^{-1} f
$$

Ce qui montre que l'opérateur $L$ est auto-adjoint sur $L^{2}\left(\mathbb{R}^{n}, \varphi^{-2} d x\right)$. On déduit aussi de (11) que

$$
(L f, f)_{L^{2}\left(\mathbb{R}^{n}, \varphi^{-2} d x\right)} \geq \lambda\|f\|_{L^{2}\left(\mathbb{R}^{n}, \varphi^{-2} d x\right)} .
$$

On a aussi

$$
\mathcal{L}_{\theta} f=\varphi(\Delta+(1-\theta) \lambda) \varphi^{-1} f
$$

et par conséquent l'opérateur $\mathcal{L}_{\theta}$ vérifie

$$
\left(\mathcal{L}_{\theta} f, f\right)_{L^{2}\left(\mathbb{R}^{n}, \varphi^{-2} d x\right)} \geq(1-\theta) \lambda\|f\|_{L^{2}\left(\mathbb{R}^{n}, \varphi^{-2} d x\right)} .
$$

Dans le cas général d'un groupe de Lie muni d'un sous-Laplacien avec "Drift" vérifiant l'hypothèse $(\mathrm{H})$ on a la relation de conjugaison

$$
\mathcal{L}_{\theta} f=\chi(\Delta+(1-\theta) \lambda) \chi^{-1} f .
$$


III. 2. Nous aurons besoin des deux ingrédients suivants:

Lemme 1. Soit $G$ un groupe de Lie connexe. Soit $\chi$ un caractère sur $G$ et $X$ un champ de vecteurs invariant à gauche tel que $X(\chi) \not \equiv 0$. Soit $1 \leq p<\infty$. Alors, il existe $C>0$

$$
\int|f(x)|^{p} \chi(x) d^{r} x \leq C \int|X f(x)|^{p} \chi(x) d^{r} x, \quad f \in C_{0}^{\infty}(G),
$$

où $d^{r} x$ est la mesure de Haar invariante à droite sur $G$.

Lemme 2. Soit $G$ un groupe de Lie connexe moyennable et $\Delta=-\sum X_{j}^{2}$ un sousLaplacien invariant à gauche sur $G$ et soit $\chi$ un caractère sur $G$ tel que $\Delta(\chi)=\lambda_{\chi}$. Alors

$$
\begin{aligned}
& \left\|\exp \left(-t \chi(L+(1-\theta) \lambda) \chi^{-1}\right)\right\|_{L^{p}\left(G, \chi^{-2} d^{r} x\right) \rightarrow L^{p}\left(G, \chi^{-2} d^{r} x\right)} \\
& \quad=\exp \left(\left[\left(1-\frac{2}{p}\right)^{2}-(1-\theta)\right] \lambda t\right)
\end{aligned}
$$

La preuve du Lemme 1est une adaptation facile de la proposition IX.2.1 de [12]. Pour la preuve du Lemme 2 notons $T_{t}$ le semi-groupe engendré par $\Delta$ et $\phi_{t}$ son noyau, i.e. le noyau donné par (cf. [12])

$$
T_{t} f(x)=\int \phi_{t}\left(y^{-1} x\right) f(y) d y, \quad f \in C_{0}^{\infty}(G) .
$$

Alors

$$
\begin{aligned}
\exp \left(-t \chi(L+(1-\theta) \lambda) \chi^{-1}\right) f(x) & =\exp (-(1-\theta) \lambda t) \chi T_{t} \chi^{-1} f(x) \\
& =\exp (-(1-\theta) \lambda t) \int\left(\chi \phi_{t}\right)\left(y^{-1} x\right) f(y) d y \\
& =\exp (-(1-\theta) \lambda t) f *\left(\chi \phi_{t}(g) d^{r} g\right) .
\end{aligned}
$$

D'où

$$
\begin{aligned}
& \left\|\exp \left(-t \chi(L+(1-\theta) \lambda) \chi^{-1}\right)\right\|_{L^{p}\left(\chi^{-2} d^{r} x\right) \rightarrow L^{p}\left(\chi^{-2} d^{r} x\right)} \\
& \quad=\exp (-(1-\theta) \lambda t) \sup _{f \neq 0} \frac{\left\|\chi^{-2 / p}\left[f * \chi \phi_{t} d^{r} g\right]\right\|_{L^{p}\left(G, d^{r} g\right)}}{\|f\|_{L^{p}\left(G, d^{r} g\right)}} \\
& \quad=\exp (-(1-\theta) \lambda t) \sup _{f \neq 0} \frac{\left\|\left(\chi^{-2 / p} f\right) *\left(\chi^{-2 / p+1} \phi_{t} d^{r} g\right)\right\|_{L^{p}\left(G, d^{r} g\right)}}{\|f\|_{L^{p}\left(G, d^{r} g\right)}} \\
& \quad=\exp (-(1-\theta) \lambda t) \int \chi^{-2 / p+1}(g) \phi_{t}(g) d^{r} g
\end{aligned}
$$


du fait de la moyennabilité de $G$ (cf. [10]). D'où en effectuant le changement de variable $g \longleftrightarrow g^{-1}$

$$
\begin{aligned}
\| \exp & \left(-t \chi(L+(1-\theta) \lambda) \chi^{-1}\right) \|_{L^{p}\left(\chi^{-2} d^{r} x\right) \rightarrow L^{p}\left(\chi^{-2} d^{r} x\right)} \\
& =\exp (-(1-\theta) \lambda t) \int \chi^{2 / p-1}(g) \phi_{t}\left(g^{-1}\right) d g \\
& =\exp (-(1-\theta) \lambda t) T_{t}\left(\chi^{2 / p-1}\right)(e) \\
& =\exp (-(1-\theta) \lambda t) \exp \left(t\left(\frac{2}{p}-1\right)^{2} \lambda\right) \\
& =\exp \left(\left[\left(\frac{2}{p}-1\right)^{2}-(1-\theta)\right] \lambda t\right) .
\end{aligned}
$$

Ce qui achève la preuve du Lemme 2 .

III. 3. Maintenant notons $T_{t}^{\theta}=\exp \left(-t \mathcal{L}_{\theta}\right), t>0$, le semi-groupe engendré par $\mathcal{L}_{\theta}$. On alors (cf. [6])

$$
\mathcal{L}_{\theta}^{-k / 2}=c \int_{0}^{\infty} t^{k / 2} T_{t}^{\theta} \frac{d t}{t}, \quad k=1,2, \ldots
$$

où $c=\Gamma(k / 2)^{-1}$. La transformée de Riesz multiple $\mathcal{R}_{k}^{\theta}=X_{i_{1}} \ldots X_{i_{k}} \mathcal{L}_{\theta}^{-k / 2}, 1 \leq$ $i_{1}, \ldots, i_{k} \leq n$, que nous noterons $\mathcal{R}_{k}^{\theta}=X_{1} \cdots X_{k} \mathcal{L}_{\theta}^{-k / 2}$ afin d'alléger les notations, peut être décomposée de la manière suivante:

$$
\begin{aligned}
\mathcal{R}_{k}^{\theta} & =c \int_{0}^{1} t^{k / 2} X_{1} \ldots X_{k} T_{t}^{\theta} \frac{d t}{t}+c \int_{1}^{\infty} t^{k / 2} X_{1} \ldots X_{k} T_{t}^{\theta} \frac{d t}{t} \\
& =\mathcal{R}_{k, 0}^{\theta}+\mathcal{R}_{k, \infty}^{\theta},
\end{aligned}
$$

et si on note $\phi_{t}^{\theta}(\cdot)$ le noyau associé à $T_{t}^{\theta}$, on a, par le principe de Harnack local parabolique (cf. [12])

$$
\left|X_{1} \ldots X_{k} \phi_{t}^{\theta}(g)\right| \leq C \phi_{t+1}^{\theta}(g), \quad t \geq 1, g \in G .
$$

D'où

$$
\left\|\mathcal{R}_{k, \infty}^{\theta}\right\|_{L^{p}\left(\chi^{-2} d^{r} x\right) \longrightarrow L^{p}\left(\chi^{-2} d^{r} x\right)} \leq C \int_{1}^{\infty} t^{k / 2-1}\left\|T_{t}^{\theta}\right\|_{L^{p}\left(\chi^{-2} d^{r} x\right) \longrightarrow L^{p}\left(\chi^{-2} d^{r} x\right)} d t .
$$

En utilisant la relation de conjugaison (2) et le lemme 2 déduit alors que

$$
\left\|\mathcal{R}_{k, \infty}^{\theta}\right\|_{L^{p}\left(\chi^{-2} d^{r} x\right) \longrightarrow L^{p}\left(\chi^{-2} d^{r} x\right)}<\infty
$$

dès que $(1-2 / p)^{2}-(1-\theta)<0$, i.e., dès que

$$
\frac{2}{1+\sqrt{1-\theta}}<p<\frac{2}{1-\sqrt{1+\theta}}
$$

dans le cas où $0<\theta<1$, et dès que $p>1$ dans le cas où $\theta=0$. 
III. 4. La partie locale $\mathcal{R}_{k, 0}^{\theta}$ est bornée sur $L^{p}\left(\chi^{-2} d^{r} x\right)$ pour tout $1<p<\infty$. En effet, soit $A>0$, écrivons

$$
\begin{aligned}
R_{0}= & c \int_{0}^{1} t^{k / 2-1} X_{1} \ldots X_{k} \exp \left(-t\left(\mathcal{L}_{\theta}+A I\right)\right) d t \\
& +c \int_{0}^{1} t^{k / 2-1} X_{1} \ldots X_{k}\left(\exp \left(-t \mathcal{L}_{\theta}\right)-\exp \left(-t\left(\mathcal{L}_{\theta}+A I\right)\right)\right) d t \\
= & c \int_{0}^{\infty} t^{k / 2-1} X_{1} \ldots X_{k} \exp \left(-t\left(\mathcal{L}_{\theta}+A I\right)\right) d t \\
& -c \int_{1}^{\infty} t^{k / 2-1} X_{1} \ldots X_{k} \exp \left(-t\left(\mathcal{L}_{\theta}+A I\right)\right) d t \\
& +c \int_{0}^{1} t^{k / 2-1} X_{1} \ldots X_{k}\left(\left(\exp \left(-t \mathcal{L}_{\theta}\right)-\exp \left(-t\left(\mathcal{L}_{\theta}+A I\right)\right)\right) d t\right. \\
= & -c \int_{1}^{\infty} t^{k / 2-1} X_{1} \ldots X_{k} \exp \left(-t\left(\mathcal{L}_{\theta}+A I\right)\right) d t \\
& +X_{1} \ldots X_{k}\left(\mathcal{L}_{\theta}+A I\right)^{-k / 2} \\
& +c \int_{0}^{1} t^{k / 2-1} X_{1} \ldots X_{k}\left(\exp \left(-t \mathcal{L}_{\theta}\right) \exp \left(-t\left(\mathcal{L}_{\theta}+A I\right)\right)\right) d t \\
= & I+I I+I I I .
\end{aligned}
$$

La bornitude de l'opérateur $I$ résulte du principe de Harnack parabolique (qui permet d'estimer le noyau $\left|X_{1} \ldots X_{k} \phi_{t}^{\theta}(\cdot)\right|$ par $\phi_{t+1}^{\theta}(\cdot), t>1$ ) et de la présence du facteur $\exp (-A t)$ qui permet pour un $A$ assez grand d'absorber la croîssance exponentielle de la norme $\left\|T_{t}^{\theta}\right\|_{L^{p}\left(\chi^{-2} d^{r} x\right) \longrightarrow L^{p}\left(\chi^{-2} d^{r} x\right)}$ et de faire converger l'intégrale. La bornitude de la transformée de Riesz $I I=X_{1} \ldots X_{k}(A I+\Delta)^{-k / 2}$, pour $A$ est assez grand, découle de faits bien connus (cf. 77, [11]). Enfin le noyau de $X_{1} \ldots X_{k}\left(\left(\exp -t\left(\mathcal{L}_{\theta}\right)-\exp \left(-t\left(\mathcal{L}_{\theta}+A I\right)\right)\right.\right.$ est donné par

$$
\left(1-e^{-A t}\right) X_{1} \ldots X_{k} \phi_{t}^{\theta} \sim t X_{1} \ldots X_{k} \phi_{t}^{\theta}
$$

et par le Théorème V.4.2 de [12], il existe $C>0$ et $c>0$ telles que

$$
\left|X_{1} \ldots X_{k} \phi_{t}^{\theta}(g)\right| \leq C t^{-k / 2} \phi_{c t}^{\theta}(g), \quad \forall g \in G, \forall 0<t<1 .
$$

En utilisant (3) et (4) on déduit facilement la bornitude de $I I I$.

III. 5. Supposons maintenant que les champs $X_{j}$ sont tels que $X_{j}(\chi) \not \equiv 0$ (i.e. $c_{j} \neq 0$ avec les notations du $\left.\S \mathrm{II}\right)$. Alors, si $\theta \neq 0$ et si $p \geq 2 /(1-\sqrt{1-\theta})$ ou $p \leq$ $2 /(1+\sqrt{1-\theta})$ la bornitude de la transformée de Riesz $\mathcal{R}_{k}^{\theta}$ entraînerait l'existence d'une constante $C>0$ telle que

$$
\left\|\mathcal{R}_{k}^{\theta} f\right\|_{L^{p}\left(\chi^{-2} d^{r} x\right)}=\left\|X_{1} \ldots X_{k} \mathcal{L}_{\theta}^{-k / 2} f\right\|_{L^{p}\left(\chi^{-2} d^{r} x\right)} \leq C\|f\|_{L^{p}\left(\chi^{-2} d^{r} x\right)} .
$$

Mais, par le lemme1, l'estimation (5) implique que

$$
\left\|X_{1} \ldots X_{k} f\right\|_{L^{p}\left(\chi^{-2} d^{r} x\right)} \leq\|f\|_{L^{p}\left(\chi^{-2} d^{r} x\right)} \leq\left\|\mathcal{L}_{\theta}^{k / 2} f\right\|_{L^{p}\left(\chi^{-2} d^{r} x\right)}
$$

et donc que

$$
\mathcal{L}_{\theta}^{-k / 2}=c \int_{0}^{\infty} t^{k / 2-1} T_{t}^{\theta} d t: L^{p}\left(G, \chi^{-2} d^{r} x\right) \longrightarrow L^{p}\left(G, \chi^{-2} d^{r} x\right),
$$


ce qui est incompatible avec le fait que

$$
\left\|T_{t}^{\theta}\right\|_{L^{p}\left(G, \chi^{-2} d^{r} x\right) \longrightarrow L^{p}\left(G, \chi^{-2} d^{r} x\right)}=\exp \left(\left[\left(\frac{2}{p}-1\right)^{2}-(1-\theta)\right] \lambda t\right) \geq 1,
$$

pour tout $t>0$ dès que $p \geq 2 /(1-\sqrt{1-\theta})$ ou $p \leq 2 /(1+\sqrt{1-\theta})$.

III. 6. La première partie de l'assertion (i) du théorème2 2 et l'assertion (ii) résultent immédiatement de (III.3) et (III.4). La deuxième partie de l'assertion (i) et l'assertion (iii) résultent de (III.5).

\section{EXEMPLES}

Le cas du groupe de Heisenberg $\mathbf{H}_{n}$. Considérons dans $\mathbf{H}_{n}$-le groupe de Heisenberg de dimension $2 n+1$-les champs de vecteurs invariants à gauche définis par

$$
\begin{aligned}
X_{j} & =\frac{\partial}{\partial x_{j}}-\frac{1}{2} y_{j} \frac{\partial}{\partial u}, \\
Y_{j} & =\frac{\partial}{\partial y_{j}}+\frac{1}{2} x_{j} \frac{\partial}{\partial u},
\end{aligned}
$$

$1 \leq j \leq n$, les coordonnées sur $\mathbf{H}_{n}$ étant données par $(x, y, u) \in \mathbf{R}^{n} \times \mathbf{R}^{n} \times \mathbf{R}$. Soit $L$ le sous-Laplacien avec Drift défini par

$$
L=-\left(\sum_{j=1}^{n} X_{j}^{2}+Y_{j}^{2}\right)+\sum_{j=1}^{n} a_{j} X_{j}+b_{j} Y_{j}
$$

où $a_{j}, b_{j} \in \mathbf{R}$. Il est facile de voir que l'opérateur $L$ vérifie l'hypothèse $(\mathrm{H})$ sur $\mathbf{H}_{n}$ pour le caractère $\chi$ définit par

$$
\chi(g)=\chi(x, y, u)=\exp \left[\frac{1}{2} \sum_{j=1}^{n}\left(a_{j} x_{j}+b_{j} y_{j}\right)\right], \quad g=(x, y, u) \in \mathbf{H}_{n},
$$

et

$$
\lambda=\frac{1}{4} \sum_{j=1}^{n}\left(a_{j}^{2}+b_{j}^{2}\right) .
$$

Le cas des groupes NA. Il s'agit d'une classe de groupes résolubles non-unimodulaires généralisant les groupes $N A$ associés aux espaces symétriques de rang 1 (cf. [5]). Rappelons que si $\mathcal{N}$ est une algèbre de Lie réele nilpotente de rang 2 munie d'un produit scalaire $(\cdot \mid \cdot)$ et si $\mathcal{Z}$ est son centre et $\mathcal{B}=\mathcal{Z}^{\perp}$ alors $\mathcal{N}$ est dite de type $(\mathrm{H})$ si pour tout $X \in \mathcal{B}$ tel que $|X|=1, \operatorname{ad}(X)$ est une isométrie surjective de $\operatorname{Ker}(\operatorname{ad}(X))^{\perp} \longrightarrow \mathcal{Z}$. Notons $N$ le groupe de Lie simplement connexe correspondant à $\mathcal{N}$. L'application exponentielle permet de paramétrer les éléments de $N=\exp (\mathcal{N})$ $\operatorname{par}(X, Z) \in \mathcal{B} \oplus \mathcal{Z}$ de sorte que

$$
(X, Z)\left(X^{\prime}, Z^{\prime}\right)=\left(X+X^{\prime}, Z+Z^{\prime}+1 / 2\left[X, X^{\prime}\right]\right) .
$$

On note $m=\operatorname{dim}(\mathcal{B}), k=\operatorname{dim}(\mathcal{Z})$ et $Q=m / 2+k$ la dimension homogène de $N$. Posons $A=\mathbb{R}^{+}$et considérons l'action de $A$ sur $N$ définie par: $(X, Z) \longrightarrow$ 
( $\left.a^{1 / 2} X, a Z\right), a \in A$. On note $S$ le produit semi-direct $N A$ associé à cette action. $S$ est une extension résoluble de $N$. Le produit de deux éléments de $S$ est donné par

$$
(X, Z, a)\left(X^{\prime}, Z^{\prime}, a^{\prime}\right)=\left(X+a^{1 / 2} X^{\prime}, Z+a Z^{\prime}+1 / 2 a^{1 / 2}\left[X, X^{\prime}\right], a a^{\prime}\right),
$$

pour tout $(X, Z, a),\left(X^{\prime}, Z^{\prime}, a^{\prime}\right) \in S$. Sur $\mathcal{S}=\mathcal{B} \oplus \mathcal{Z} \oplus \mathbb{R}$ (l'algèbre de Lie de $S$ ) on considère le produit scalaire

$$
\left((X, Z, t) \mid\left(X^{\prime}, Z^{\prime}, t^{\prime}\right)\right)=\left(X \mid X^{\prime}\right)+\left(Z \mid Z^{\prime}\right)+t t^{\prime} .
$$

La mesure de Haar invariante à gauche (resp.: à droite) sur $S$ est donnée par $d^{l} x=d x=a^{-Q-1} d X d Z d a$ (resp.: $d^{r} x=a^{-1} d X d Z d a$ ). Le groupe $S$ est non unimodulaire et la fonction module $\delta$ est donnée par $\delta(X, Z, a)=d^{l} x / d^{r} x=a^{-Q}$.

Soient $e_{1}, \ldots, e_{m} \in \mathcal{B}, e_{m+1}, \ldots, e_{m+k} \in \mathcal{Z}, e_{0} \in \mathbb{R}$ formant une base orthonormée de $\mathcal{S}=\mathcal{B} \oplus \mathcal{Z} \oplus \mathbb{R}$. On note $X_{j}, j=0, \ldots, m+k$, les champs de vecteurs invariants à gauche définis par les vecteurs $e_{j}$ et $L$ l'opérateur défini par

$$
L=\Delta+\alpha X_{0}=-\left(X_{0}^{2}+X_{1}^{2}+\cdots+X_{m+k}^{2}\right)+\alpha X_{0}
$$

où $0 \neq \alpha \in \mathbb{R}$.

En utilisant le fait que les champs $X_{1}, \ldots, X_{m+k}$ ne contiennent pas de dérivation en $a$ et que $X_{0}=a \partial_{a}$ (cf. 5) on voit facilement que l'opérateur $L$ défini par (6) vérifie l'hypothèse (H) sur le groupe $S$ avec $\chi=a^{\alpha / 2}$ et $\lambda=\alpha^{2} / 4$.

Remarquons que si $\alpha=Q$, alors $L$ coincide, au signe près, avec l'opérateur de Laplace-Beltrami associé à la métrique riemannienne invariante à gauche induite par le produit scalaire sur $\mathcal{S}$ et l'espace $L^{p}\left(S, a^{-\alpha} d^{r} g\right)=L^{p}\left(S, a^{-Q} d^{r} g\right)=L^{p}\left(S, d^{l} g\right)$ est l'espace de Lebesgue associé à la mesure de Haar invariante à gauche sur $S$. Let résultat (ii) du théorème 2 est dans ce cas contenu dans [3].

\section{REFERENCES}

[1] G. Alexopoulos, An application of homogenization theory to harmonic analysis: Harnack inequalities and Riesz transforms on Lie groups of polynomial growth, Canad. J. Math. 44 (1992), 691-727. MR 93j:22006

[2] G. Alexopoulos, Puissances de convolution sur les groupes à croissance polynomiale du volume, C. R. Acad. Sci. Série I 324 (1997), 771-776. MR 98a:43001

[3] J.-P. Anker, E. Damek, C. Yacoub, Spherical analysis on harmonic AN groups, Ann. Scuola Norm. Sup. Pisa Cl. Sci. (4) 23 (1996), 643-679. MR 99a:22014

[4] R. R. Coifman et G. Weiss, Analyse harmonique non commutative sur certains espaces homogènes, Lecture Notes in Math., vol. 242, Springer-Verlag, 1971. MR 58:17690

[5] E. Damek et F. Ricci, Harmonic analysis on solvable extensions of $H$-type groups, J. Geom. Anal. 3 (1992), 213-248. MR 93d:43006

[6] B. M. Davies, One-parameter semigroups, Academic Press, 1980. MR 82i:47060

[7] N. Lohoué, Transformées de Riesz et fonctions de Littlewood Paley sur les groupes non moyennables, C. R. Acad. Sci. Série I 306 (1988), 327-330. MR 89b:43008

[8] N. Lohoué, Transformées de Riesz et fonctions sommables, Amer. J. Math. 114 (1992), 875-992. MR 93k:58214

[9] N. Lohoué et S. Mustapha, Sur les transformées de Riesz sur les groupes de Lie moyennables et sur certains espaces homogènes, Canad. J. Math. 50 (1998), 1090-1104. MR 2000a:43008

[10] H. Reiter, Classical Harmonic Analysis and Locally Compact Groups, Oxford Math. Monograph, 1968. MR 46:5933

[11] D. W. Robinson, Elliptic Operators and Lie Groups, Clarendon Press, Oxford, New York, Tokyo, 1991. MR 92m:58133 
[12] N. Th. Varopoulos, L. Saloff-Coste et Th. Coulhon, Analysis and Geometry on Groups, Cambridge Tracts in Math., 100, 1993. MR 95f:43008

[13] K. Yosida, Functional Analysis, Springer-Verlag, 1978. MR 58:17765

Departement de Mathématiques, Bât. 425, Université Paris Xi, 91405, Orsay Cedex, FRANCE

Institut de Mathématiques, Université Paris Vi, 4, Place Jussieu, 75252, Paris Cedex, FRANCE 